\title{
Mucociliary function, ciliary ultrastructure, and ciliary orientation in Young's syndrome
}

\author{
Robbert de Iongh, Alvin Ing, Jonathan Rutland
}

\begin{abstract}
Background Mucociliary clearance is impaired in patients with Young's syndrome (obstructive azoospermia with recurrent sinobronchial disease), cystic fibrosis, and primary ciliary dyskinesia. No defect of cilia or mucus has been detected in Young's syndrome.

Methods Ciliary function and ultrastructure, including ciliary orientation, were studied quantitatively in 20 patients with Young's syndrome and 20 normal subjects to determine the incidences of ciliary defects. Nasal ciliated epithelium was obtained from each subject and used for measurement of ciliary beat frequency and ultrastructural analyses. Ciliary orientation was determined by measuring ciliary deviation in electron micrographs; ciliary deviation is a measure of the relative orientation of cilia in relation to each other in which high values indicate ciliary disorientation.

Results Ciliary beat frequency and the incidence of microtubular defects and numbers of dynein arms did not differ between patients with Young's syndrome and control subjects. In patients with Young's syndrome basal ciliary deviation $\left(16.0^{\circ}\right)$ was similar to that in control subjects $\left(14 \cdot 1^{\circ}\right)$, but at the ciliary tip ciliary deviation $\left(21.9^{\circ}\right)$ was greater than in healthy subjects $\left(14 \cdot 5^{\circ}\right)$.

Conclusion The relative disorientation of the distal ciliary axoneme in patients with Young's syndrome compared with normal subjects may be due to a structural defect but is more likely to be a consequence of abnormal mucus.
\end{abstract}

The association between male sterility and recurrent respiratory tract infection is common to cystic fibrosis, ${ }^{1}$ primary ciliary dyskinesia, ${ }^{2}$ and Young's syndrome. ${ }^{3}$ Young described 52 men with obstructive azoospermia, of whom over half "had had a defect in their lungs," including bronchitis from childhood and bronchiectasis. $^{3}$ No underlying cause has been established for the respiratory disease in such patients or the association with obstructive azoospermia.

Mucociliary clearance is an important defence mechanism in the respiratory tract and alteration of either of its two main components (ciliary activity or mucus) may result in recurrent respiratory tract disease. In cystic fibrosis abnormal mucus secretion is believed to cause impaired mucociliary clearance and hence recurrent respiratory tract infection. ${ }^{1}$ Azoospermia in male patients with cystic fibrosis is due to atresia or malformation of the vasa and epididymis. ${ }^{4}$ In primary ciliary dyskinesia impaired mucociliary clearance has been shown to be due to structural defects of the ciliary axoneme. ${ }^{5-9}$ Similar structural defects of the sperm tail axoneme in these patients result in immotile or poorly motile sperm. In patients with Young's syndrome nasal and tracheobronchial mucociliary clearance is impaired or absent. ${ }^{1011}$ No underlying mechanism has been identified to explain either the respiratory problems or the obstructive azoospermia. Most studies of Young's syndrome report that cilia and sperm have normal axonemal ultrastructure, ${ }^{11-14}$ although microtubular abnormalities, ${ }^{1516}$ compound cilia, ${ }^{16}$ and reduced numbers of inner dynein arms $^{15}$ have been reported in individual patients. Abnormal ciliary orientation has been described in two patients but not quantified. ${ }^{12}$

We report a quantitative ultrastructural analysis of nasal ciliated epithelium in 20 patients with Young's syndrome compared with a group of 20 healthy subjects matched for age and smoking habits. In particular we examined the orientation of cilia with a recently developed computer aided technique. ${ }^{17}$

\section{Patients and methods}

Twenty patients with azoospermia (mean age 44 (SD 12) and a history of recurrent lower respiratory tract infection or sinusitis, or both, were studied. All patients had a clinical interview, sinus and chest radiography, and physical examination. Primary ciliary dyskinesia was excluded by measurement of ciliary beat frequency and ciliary ultrastructure. None of the patients had a family history of cystic fibrosis. These patients did not have sweat tests, and those in whom the possibility of cystic fibrosis was considered were excluded from this study. Apart from one patient whose brother also had features of Young's syndrome, none of the patients had a family history of respiratory tract infection. None of the patients had any gastrointestinal or pancreatic manifestations of cystic fibrosis. Obstructive azoospermia was documented in all patients, and eight patients had testicular biopsies showing normal sperm maturation. Two patients had bilateral microsurgical epididymovasostomies, which were successful in one. At the time of investigation none of the patients had current or recent
Address for correspondence: Mr de Iongh

Accepted 22 November 1991 
Table 1 Respiratory function in patients with Young's syndrome $(n=14)$. All values are percentages of predicted values in an Australian population

\begin{tabular}{lllllll}
\hline & $F E V_{1}$ & $F V C$ & $F R C$ & $R V$ & $T L C$ & $T L C O$ \\
\hline Mean & $80 \cdot 4$ & $91 \cdot 6$ & $83 \cdot 9$ & 105 & $93 \cdot 6$ & $85 \cdot 3$ \\
SD & $15 \cdot 4$ & $15 \cdot 2$ & $15 \cdot 7$ & 29 & $12 \cdot 6$ & $13 \cdot 6$ \\
Range & $54-108$ & $77-126$ & $48-106$ & $66-186$ & $76-118$ & $64-105$ \\
\hline
\end{tabular}

$\mathrm{FEV}_{1}$ = forced expiratory volume in one second; FVC = forced vital capacity; FRC = functional residual capacity; RV = residual volume; TLC = total lung capacity; TLCo = carbon monoxide transfer factor.

respiratory tract infections (either bacterial or viral).

Chest radiographs in 16 patients showed abnormalities in 13 , including cystic changes, bronchiectasis, calcified lymph nodes and pleural shadows. Radiographs of the paranasal sinuses were abnormal in 11 of the 16 patients examined. Changes included mucosal thickening, fluid levels, and polyps. Results of respiratory function tests are listed in table 1 .

Twenty volunteers (mean age $42(12)$ ) with no history of recurrent respiratory tract disease were also studied. They were matched with the patients for age and smoking habits (table 2). The study was approved by the ethics committee at Concord Hospital, and all subjects gave informed consent.

\section{INVESTIGATIONS}

Nasal mucociliary clearance time was measured by the saccharin test. ${ }^{18}$ When no clearance was demonstrated after one hour a value of 60 minutes was assigned for calculation of the group mean clearance time. Nasal mucociliary clearance time was not measured in control subjects, as a previous study established that nasal mucociliary clearance time by this technique is less than 30 minutes in normal subjects. ${ }^{18}$ Respiratory function tests were performed in only 14 patients.

Samples of nasal ciliated epithelium were obtained by nasal mucosal brushing ${ }^{19}{ }^{20}$ from all subjects for photometric determination ${ }^{19}$ of ciliary beat frequencies and for electron microscopy. ${ }^{1720}$ The following features of ciliary ultrastructure were analysed quantitatively from electron micrographs of transversely sectioned cilia: the incidence of compound cilia, the incidence of central and peripheral microtubule defects, the numbers of inner and outer dynein arms per cilium, and ciliary orientation. Peripheral tubule defects were defined as missing or displaced peripheral

Table 2 Smoking history and mucociliary function in patients with Young's syndrome

\begin{tabular}{|c|c|c|c|c|}
\hline & Age (years) & Pack years & $N M C C(\min )$ & $C B F(H z)$ \\
\hline \multicolumn{5}{|c|}{ Normal subjects $(n=20)$} \\
\hline $\begin{array}{l}\text { Mean } \\
\text { SD } \\
\text { Range }\end{array}$ & $\begin{array}{l}44 \\
12 \\
28-68\end{array}$ & $\begin{array}{l}10 \cdot 7 \\
16 \cdot 8 \\
0-50\end{array}$ & $\begin{array}{r}10^{\star} \\
2 \\
<30\end{array}$ & $\begin{array}{l}11 \cdot 6 \\
1 \cdot 0 \\
10-13 \cdot 7\end{array}$ \\
\hline \multicolumn{5}{|c|}{ Patients with Young's syndrome $(n=20)$} \\
\hline $\begin{array}{l}\text { Mean } \\
\text { SD } \\
\text { Range }\end{array}$ & $\begin{array}{l}42 \\
12 \\
29-71\end{array}$ & $\begin{array}{l}11 \cdot 7 \\
17 \cdot 9 \\
0-54\end{array}$ & $\begin{array}{l}33 \dagger \\
21 \\
9-60+\end{array}$ & $\begin{array}{l}11.9 \\
1.3 \\
10 \cdot 2-15 \cdot 1\end{array}$ \\
\hline
\end{tabular}

NMCC = nasal mucociliary clearance time; CBF = ciliary beat frequency. $\star$ Data from Rutland and Cole. ${ }^{18}$

†Underestimation of true mean-an arbitrary value of 60 min was assigned for NMCC $>60 \mathrm{~min}$. doublets or extra microtubules (single or double) outside the peripheral ring of doublets. Central tubule defects included missing central microtubules or extra single microtubules within the peripheral ring of doublets. Profiles of cilia that were clearly sectioned near the tip or base of the cilium were not included in the counting procedures as the normal termination of ciliary microtubules at these levels may lead to an overestimation of abnormal cilia. The relative orientation of cilia was also measured. ${ }^{17}$ The ciliary beat axis is perpendicular to a line drawn through the centres of the two central microtubules (figure 1). The angle subtended by this line to an arbitrary reference line was measured from electron micrographs with a graphics tablet interfaced to a computer. ${ }^{17}$ The mean ciliary angle and the standard deviation (ciliary deviation) from this mean angle were determined. The ciliary deviation provides a measure of the variation in the ciliary beat axis. Ciliary deviation was measured at two levels: near the tips and near the bases of the cilia. In transverse sections of cilia the presence of microvilli indicates a plane of section near the bases of the cilia and the absence of microvilli

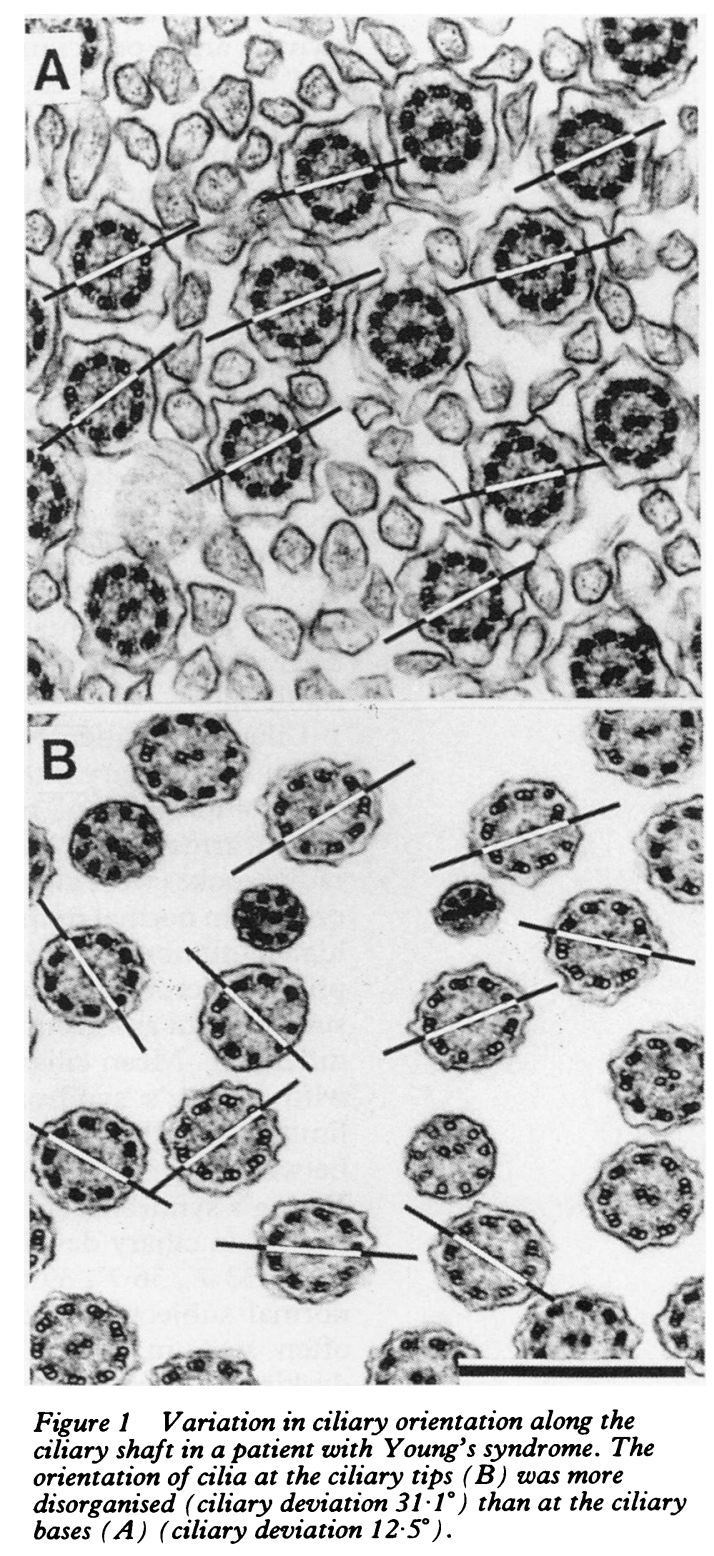


Table 3 Ciliary ultrastructure in patients with Young's syndrome

\begin{tabular}{|c|c|c|c|c|c|c|c|c|}
\hline & \multirow[b]{2}{*}{$C C(\%)$} & \multirow[b]{2}{*}{$C T(\%)$} & \multirow[b]{2}{*}{$P T(\%)$} & \multirow[b]{2}{*}{ DAi/cilium } & \multirow[b]{2}{*}{ DAo/cilium } & \multicolumn{3}{|c|}{ Ciliary deviation (degrees) } \\
\hline & & & & & & Base & Tip & Total \\
\hline \multicolumn{9}{|c|}{ Normal subjects $(n=20)$} \\
\hline Mean & $0 \cdot 3$ & $1 \cdot 0$ & 1.8 & $5 \cdot 6$ & $8 \cdot 4$ & $14 \cdot 1$ & $14 \cdot 5$ & $14 \cdot 8$ \\
\hline SD & 0.5 & 1.8 & $1 \cdot 3$ & $1 \cdot 1$ & & $4 \cdot 2$ & 3.5 & $4 \cdot 1$ \\
\hline Range & $0-1 \cdot 6$ & $0-7 \cdot 8$ & $0-5 \cdot 0$ & $3 \cdot 7-8 \cdot 0$ & $7.6-8.9$ & $7 \cdot 4-26 \cdot 7$ & $9 \cdot 3-23 \cdot 9$ & $9 \cdot 7-24$ \\
\hline \multicolumn{9}{|c|}{ Patients with Young's syndrome $(n=20)$} \\
\hline Mean & $1 \cdot 4$ & $1 \cdot 7$ & $3 \cdot 3$ & $5 \cdot 7$ & 8.5 & $16 \cdot 6$ & $21.9 \star$ & $20 \cdot 4^{\star}$ \\
\hline SD & $2 \cdot 0$ & 1.8 & $4 \cdot 8$ & $1 \cdot 1$ & 0.5 & $7 \cdot 5$ & $8 \cdot 7$ & $7 \cdot 0$ \\
\hline Range & $0-7.5$ & $0-6 \cdot 6$ & $0-21 \cdot 5$ & $3 \cdot 7-7 \cdot 6$ & $7 \cdot 0-9 \cdot 0$ & $9 \cdot 5-21 \cdot 4$ & $10 \cdot 4-40 \cdot 1$ & $11 \cdot 7-33 \cdot 4$ \\
\hline
\end{tabular}

${ }^{\star} \mathrm{p}<0.01$ ( $t$ test)

$\mathrm{CC}=$ compound cilia; $\mathrm{CT}=$ central tubule defects; $\mathrm{PT}=$ peripheral tubule defects; $\mathrm{DAi}=$ inner dynein arm numbers; DAo = outer dynein arm numbers.

indicates a plane of section nearer the tips. After separate analyses of ciliary deviation at these two levels data were pooled to provide a total measure of ciliary deviation for each patient.

\section{ANALYSIS}

When data were distributed normally (nasal mucociliary clearance time, ciliary beat frequency, ciliary deviation, and numbers of dynein arms per cilium) analysis of variance and $t$ tests were used. The incidence of compound cilia and of central and peripheral microtubule defects and the numbers of inner and outer dynein arms per cilium were compared by the Mann-Whitney U test.

\section{Results}

Mean nasal mucociliary clearance time in Young's syndrome patients was significantly prolonged $(p<0.001)$ compared with normal values. Mean ciliary beat frequency was not significantly different $(p>0.05)$ from normal subjects. Ciliary beat frequency in all patients with Young's syndrome was within normal limits. Ten patients had nasal mucociliary clearance times greater than 60 minutes (table 2).

Cilia from patients with Young's syndrome had normal ciliary ultrastructure with the normal complement of microtubules $(9+2)$ and dynein arms (table 3 ). Although not quantified, radial spokes were also visible and seemed to be present in normal numbers. Some patients had higher numbers of compound cilia and peripheral microtubule defects than had normal subjects, but as a group there was no significant difference. Mean ciliary deviation for patients with Young's syndrome was within normal limits, ${ }^{17}$ but there was a significant difference between normal subjects and patients with Young's syndrome $(p<0.01)$. Seven patients had mean ciliary deviation $\left(25 \cdot 5^{\circ}, 26 \cdot 6^{\circ}, 29 \cdot 3^{\circ}\right.$, $33.6^{\circ}, 33 \cdot 7^{\circ}, 36 \cdot 7^{\circ}, 39 \cdot 6^{\circ}$ ) outside the range for normal subjects. Random ciliary orientation, often seen in patients with primary ciliary dyskinesia, ${ }^{17}$ was not found in any patient with Young's syndrome. Comparison of ciliary deviation at two levels along the length of the axoneme showed that at the ciliary base ciliary deviation was not significantly different from that in normal subjects, although at the tips of the cilia ciliary deviation was significantly greater $(\mathrm{p}<0.01)$ than that in normal subjects. The different orientation of cilia at these two levels is shown in figure 1 . The distribution of ciliary angles in patients with Young's syndrome (figure 2) showed a small increase in the numbers of cilia with large angles of deviation from the mean angle compared with norma subjects.

\section{Discussion}

The aetiology of Young's syndrome is unknown. ${ }^{101121-24}$ Repeated studies have shown sweat electrolyte concentrations to be normal. ${ }^{14162124-26}$ We have confirmed the finding
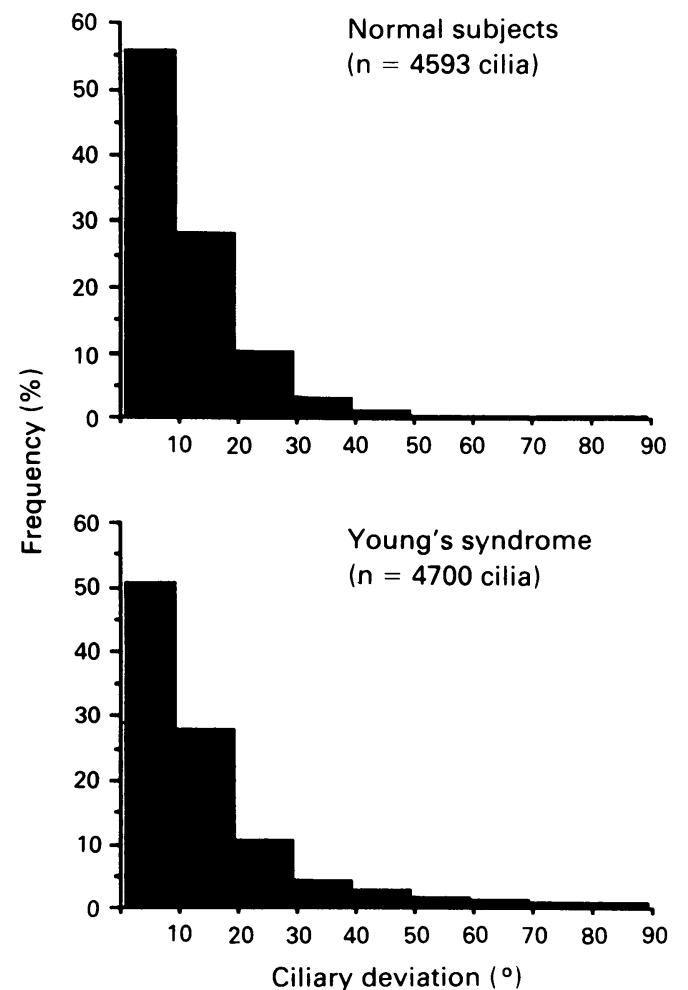

Figure 2 Pooled distribution histograms of ciliary angles of deviation in normal subjects and in patients with Young's syndrome. The distributions were similar, but the patients had slightly increased numbers of cilia with deviations from the mean angle in the range of $40-90^{\circ}$ and the frequency of deviations of less than $10^{\circ}$ was reduced by $5 \%$ 
that mucociliary clearance may be impaired but with normal ciliary beat frequency and internal ciliary ultrastructure. ${ }^{11}$ There was no evidence of reduced numbers of inner dynein arms or an increased incidence of microtubule defects as shown in case reports. ${ }^{1516}$ The increased incidence of compound cilia and the occurrence of microtubule defects that we found confirm a previous report, ${ }^{16}$ although these defects are unlikely to be a primary defect as they occur in normal subjects and as non-specific defects after respiratory tract infection. ${ }^{27-31}$

Abnormal ciliary orientation has been described previously in Young's syndrome but has not been quantified. ${ }^{12}$ Ciliary deviation in our group of patients was significantly greater than that in normal subjects but was not as great as that seen in primary ciliary dyskinesia. ${ }^{17}$ Ciliary disorientation may be the only defect in patients with recurrent respiratory disease. ${ }^{32}$ This finding of moderate ciliary disorientation could offer a possible explanation for the reduced mucociliary clearance found in Young's syndrome. It is possible that the twisting of the cilia may be a manifestation of a subtle structural microtubule defect not detectable by electron microscopy. The inconsistency of this finding suggests, however, that this is probably an epiphenomenon and more likely to be a secondary effect of recurrent respiratory tract infection than a primary cause of the respiratory tract disease.

Young's syndrome has been proposed as being a forme fruste of cystic fibrosis that results in abnormal respiratory mucus and inspissated seminal secretions. Although Young's syndrome is probably not a variant of cystic fibrosis, because sweat electrolyte concentrations are normal, the pancreas is not affected, and the clinical picture and genital dysfunction are different, the rheological properties of mucus may be abnormal in Young's syndrome. Lopez-Vidriero et al reported the viscoelastic properties of mucus in Young's syndrome and considered them consistent with a "chronic hypersecretory defect." 33 The finding of ciliary disorientation only at the tips of cilia in our group of patients might be consistent with this suggestion of abnormal mucus. Viscid secretions may cause cilia to beat inconsistently or become trapped in the mucus layer, resulting in twisting of the distal parts of the ciliary axoneme. A mucus defect of varying severity might explain the variable occurrence of ciliary disorientation in Young's syndrome. A trial of "mucoregulatory agents" in Young's syndrome showed no effect on mucociliary clearance, sperm counts, or pulmonary function. ${ }^{34}$

We have found more variable orientation of the distal ciliary axoneme in patients with Young's syndrome than in normal subjects. Though this might be due to a subtle structural defect, it could also be a consequence of abnormal mucus.

We thank Associate Professor R Newland and Mr J R Perkins of the Department of Anatomical Pathology, Concord Hospital, for their help and access to the facilities of the electron microscopy laboratory. This work was supported by a research grant from the Department of Veterans' Affairs, Commonwealth of Australia, and the Clive and Vera Ramaciotti Foundation.
1 di Sant' Agnese PA, Davis PB. Research in cystic fibrosis. N Engl J Med 1976;295:481-5.

2 Sleigh MA. Primary ciliary dyskinesia. Lancet 1981;ii:476. Young D. Surgical treatment of male infertility. J Reprod Fertil 1970;23:541-2.

4 Holsclaw DS, Perlmutter AD, Jockin H, Shwachman H. Genital abnormalities in male patients with cystic fibrosis. J Urol 1971;106:568-74.

5 Afzelius BA. A human syndrome caused by immotile cilia. Science 1976;193:317-9.

6 Pedersen $\mathrm{H}$, Mygind N. Absence of axonemal arms in the nasal mucosal cilia in Kartagener's syndrome. Nature 1976;262:494-5.

7 Eliasson R, Mossberg B, Camner P, Afzelius BA. The immotile cilia syndrome. A congenital abnornmality as an etiologic factor in chronic airway infection and male sterility. N Engl J Med 1977;297:1-6.

8 Sturgess JM, Chao J, Wong J, Aspin N, Turner JAP. Cilia with defective radial spokes: a cause of impaired ciliary motility. N Engl J Med 1979;300:53-6.

9 Sturgess JM, Chao J, Turner JAP. Transposition of ciliary microtubules: another cause of impaired ciliary motility. $N$ Engl J Med 1980;303:318-22.

10 Pavia D, Agnew JE, Bateman JRM, Sheahan NF, Knight RK, Hendry WF, et al. Lung mucociliary clearance in patients with Young's syndrome. Chest 1981;80:892-5.

11 Greenstone MA, Rutman A, Hendry WF, Cole PJ. Ciliary function in Young's syndrome. Thorax 1988;43:153-4.

12 Hendry WF, Knight RK, Whitfield HN, Stansfield AG, Pryse-Davies J, Ryder TA, et al. Obstructive azoospermia: Respiratory function tests, electron microscopy and the results of surgery. Br J Urol 1978;50:598-604.

13 Le Lannou D, Verger JP, Kernec J, Segalen J, Lescott D, Lobel B. Structure de l'epithelium bronchique dans l'association infertile masculine et bronchiectasie. INSERM 1981;103:117-22.

14 Schanker HMJ, Rajfer J, Saxon A. Recurrent respiratory disease, azoospermia and polyposis. A syndrome that mimics cystic fibrosis and immotile cilia syndrome. Arch Intern Med 1985;145:2201-3.

15 Teichtahl H, Temple-Smith PD, Johnson JL, Southwith GJ, de Kretser DM. Obstructive azoospermia and chronic sinobronchial disease (Young's syndrome) in identical twins. Fertil Steril 1987;47:879-81.

16 Gugger M, Caduff JH, Straub PW. Young-syndrom: Chromische pulmonale infekte und infertilität. Schweiz Med Wochenschr 1984;114:620-4.

17 de Iongh R, Rutland J. Orientation of respiratory tract cilia in patients with primary ciliary dyskinesia, bronchiectasis and in normal subjects. J Clin Pathol 1989;42:613-9.

18 Rutland J, Cole PJ. Nasal mucociliary clearance and ciliary beat frequency in cystic fibrosis compared with sinusitis and bronchiectasis. Thorax 1981;36:654-8.

19 Rutland J, Cole PJ. Non-invasive sampling of nasal cilia for the measurement of beat frequency and study of ultrastructure. Lancet 1980;ii:564-5.

20 Rutland J, Dewar A, Cox T, Cole PJ. Nasal brushing for the study of ciliary ultrastructure. J Clin Pathol 1982;35: 356-9.

21 Neville E, Brewis R, Yeates WK, Burridge A. Respiratory disease and obstructive azoospermia. Thorax 1983;38: 929-33.

22 Jequier AM, Holmes SC. Aetiological factors in the production of obstructive azoospermia. Br J Urol 1984;56:540-3.

23 Jequier AM. Obstructive azoospermia: a study of 102 patients. Clinical Reproduction and Fertility 1985;3: 21-36.

24 Handelsman DJ, Conway AJ, Boylan LM, Turtle JR. Obstructive azoospermia and chronic sinopulmonary infections. N Engl J Med 1984;310:3-9.

25 Khan MA, Noah MS, Bashi SA, Joharjy A, Obaid N. Young's syndrome. European Journal of Respiratory Diseases 1987;70:62-4

26 Hughes TM, Skolnick JL, Belker AM. Young's syndrome: An often unrecognised correctable cause of obstructive azoospermia. J Urol 1987;137:1238-40.

27 Afzelius BA, Camner P, Mossberg B. Acquired ciliary defects compared to those seen in immotile-cilia syndrome. European Journal of Respiratory Diseases 1983; 64(suppl 127):5-10

28 Lungarella G, Fonzi L, Ermini G. Abnormalities of bronchial cilia in patients with chronic bronchitis: an ultrastructural and qualitative analysis. Lung 1983;161: 147-56.

29 Cornillie FJ, Lauweryns JM, Corbeel L. Atypical bronchial cilia in children with recurrent respiratory infections. Pathol Res Pract 1984;178:595-604.

30 Carson JL, Collier AM, Hu SCS. Acquired ciliary defects in nasal epithelium of children with acute viral upper respiratory infections. $N$ Engl J Med 1985;312:463-8.

31 Fontolliet C, Terrier G. Abnormalities of cilia and chronic sinusitis. Rhinology 1987;25:57-62.

32 Rutland J, de Iongh RU. Random ciliary orientation. A cause of respiratory tract disease. N Engl J Med 1990; 323:1681-4.

33 Lopez-Vidriero MT, Pavia D, Greenstone M, Hendry WF, Clarke SW. Viscoelastic properties of sputum from patients with Young's syndrome. Thorax 1986;41:254.

34 Currie DC, Greenstone M, Pavia D, Agnew JE, Pellow P, Clarke SW, et al. Efficacy of "mucoregulatory" agents in Young's syndrome. Thorax 1988;43:480-1. 